\title{
Seizure onset zone localization from ictal high-density EEG in refractory focal epilepsy
}

\author{
Willeke Staljanssens ${ }^{\mathrm{a}, \mathrm{b}, *}$, Gregor Strobbe ${ }^{\mathrm{a}, \mathrm{b}}$, Roel Van Holen ${ }^{\mathrm{a}, \mathrm{b}}$, Gwénaël \\ Birot $^{c}$, Markus Gschwind ${ }^{c, d}$, Margitta Seeck ${ }^{\mathrm{d}}$, Stefaan Vandenberghe ${ }^{\mathrm{a}, \mathrm{b}}$, Serge \\ Vulliémoz, ${ }^{\mathrm{c}, \mathrm{d}}$, Pieter van Mierlo ${ }^{\mathrm{a}, \mathrm{b}, \mathrm{c}}$ \\ ${ }^{a}$ MEDISIP, Department of Electronics and Information Systems, Ghent University, De \\ Pintelaan 185, Building B Entrance 36, 9000, Ghent, Belgium \\ ${ }^{b}$ iMinds Medical IT, Belgium \\ ${ }^{c}$ Functional Brain Mapping Laboratory, Department of Fundamental Neurosciences, \\ Campus Biotech, University of Geneva, Geneva, Switzerland \\ ${ }^{d}$ EEG and Epilepsy Unit, Neurology Department, University Hospitals and Faculty of \\ Medicine of Geneva, Geneva, Switzerland
}

\begin{abstract}
Epilepsy surgery is the most efficient treatment option for patients with refractory epilepsy. Before surgery, it is of utmost importance to accurately delineate the seizure onset zone (SOZ). Non-invasive EEG is the most used neuroimaging technique to diagnose epilepsy, but it is hard to localize the SOZ from EEG due to its low spatial resolution and because epilepsy is a network disease, with several brain regions becoming active during a seizure. In this work, we propose and validate an approach based on EEG source imaging (ESI) combined with functional connectivity analysis to overcome these problems. We considered both simulations and real data of patients. Ictal epochs of 204-channel EEG and subsets down to 32 channels were analyzed. ESI was done using realistic head models and LORETA was used as inverse technique. The connectivity pattern between the reconstructed sources was calculated, and the source with the highest number of outgoing connections was selected as SOZ. We compared this algorithm with a more straightforward approach, i.e. selecting the source with the highest power after ESI as the SOZ. We found that functional connectivity
\end{abstract}

\footnotetext{
* Corresponding author

Email address: Willeke.Staljanssens@UGent.be, +32 93324326 (Willeke Staljanssens)
}

Preprint submitted to Journal of Brain Topography

July 29, 2016 
analysis estimated the SOZ consistently closer to the simulated EZ/RZ than localization based on maximal power. Performance, however, decreased when 128 electrodes or less were used, especially in the realistic data. The results show the added value of functional connectivity analysis for SOZ localization, when the EEG is obtained with a high-density setup. Next to this, the method can potentially be used as objective tool in clinical settings.

Keywords: high-density electroencephalogram (hd-EEG), EEG source imaging (ESI), functional connectivity, Granger causality, refractory epilepsy

\section{Introduction}

The electroencephalogram (EEG) is the most important clinical technique to diagnose and characterize epilepsy, because it can directly measure the aberrant electrical activity in the brain associated with this disease in a convenient, safe,

5 and inexpensive way (Smith 2005). Patients with epilepsy have a strongly abnormal ictal EEG during seizures, but also in between seizures abnormalities such as interictal epileptiform discharges (IEDs) and slow waves can be noticed.

The goal of epilepsy treatment is to suppress seizures. When antiepileptic drugs (AEDs) do not allow seizure control, surgery is an important option due to its high efficacy in selected candidates (Téllez-Zenteno et al. 2005, de Tisi et al. 2011). During epilepsy surgery, the brain region that causes the seizures is disconnected (disconnective surgery) or removed (resective surgery). Therefore, it is of utmost importance to accurately delineate the epileptogenic zone (EZ), that is the brain area of which surgical removal is required and sufficient to render the patient seizure-free. However, the EZ is only a conceptual region since it cannot be measured directly. Only when a patient is seizure-free after surgery, we can conclude that the EZ was harbored in the resected zone (RZ) (Rosenow and Lüders 2001). Fortunately, it is possible to get an indirect estimate of the EZ by localizing the seizure onset zone (SOZ), which is the region where clinical seizures originate, and/or the irritative zone (IZ), i.e. the region where interictal epileptiform discharges (IEDs) originate. In current clinical 
practice, visual inspection of the EEG is combined with other investigations such as MRI, SPECT, PET, MEG, and invasive EEG (iEEG) with implanted electrodes, to localize these areas (Carrette et al. 2011). Yet, individually, none of these techniques are able to localize SOZ unambiguously. On the other hand, every extra investigation is expensive, time-consuming, and potentially harmful for the patient, e.g. iEEG can lead to scarring, infection and functional loss (Sprengers et al. 2014).

Therefore, it would be of high clinical value to be able to localize the SOZ based on non-invasive EEG alone, which is an inexpensive and safe technique. However, several problems are encountered when estimating the SOZ from noninvasive EEG. One of the major problems is the low spatial resolution $(\sim \mathrm{cm})$ of non-invasive EEG due to volume conduction. Neuronal activity propagates through different tissues (such as cerebrospinal fluid, skull and scalp) before it 35 reaches the electrodes. Because of the different conductivities of these tissues, and certainly the low conductivity of the skull, the measured activity at the electrodes is smeared and distorted. As a consequence, the potential measured at a given electrode does not necessarily represent the activity of the directly underlying brain area. The second major problem is that epilepsy is a network 40 disease, i.e. during a seizure, several brain regions become simultaneously active as part of the patient's individual epileptic network and it is often hard to distinguish the main driver(s) of this network at seizure onset from the secondary activated regions (Spencer 2002, Richardson 2012).

One way to tackle the problem of the low spatial resolution is to reconstruct 45 the sources in the brain that are generating the EEG using EEG source imaging (ESI) techniques. In recent years, a large body of research to measure the EZ based on ESI of EEG recordings has been done. ESI can be applied on interictal or ictal EEG measurements to get information about the IZ and the SOZ, respectively. The vast majority of studies has focused on the localization 50 of the IZ (Plummer et al. 2008, Brodbeck et al. 2011, Wennberg et al. 2011. Mégevand et al. 2014, Wennberg and Cheyne 2014, Strobbe et al. 2016). Yet, it can be argued that identification of the $\mathrm{SOZ}$ is more indicative of the $\mathrm{EZ}$ 
compared to the IZ, since they directly reflect the seizures(Jayakar et al. 1991 Rémi et al. 2011, Elshoff et al. 2013).

However, ESI of ictal activity is significantly harder to obtain due to muscle and movement artifacts during seizures. Despite these difficulties, some methods analyzing ictal EEG recordings have been proposed, suggesting that ESI of jictal activity is promising for SOZ localization (Lantz et al. 1999, Assaf and Ebersole 1997, Ebersole 2000, Boon et al. 2002; Jung et al. 2009, Koessler 60 et al. 2010; Yang et al. 2011; Habib et al. 2015).

ESI can be improved by increasing the spatial resolution of the EEG by including more electrodes. Despite the fact that increasing the number of electrodes does not solve the distortion of the brain signals, previous research has shown the benefit of high-density EEG (hd-EEG) on ESI (Lantz et al. 2003

65 Michel and Murray 2012), with specificity and sensitivity increasing significantly with the number of electrodes used (Brodbeck et al. 2011).

The aforementioned studies did not take into account that epilepsy is a network disease and thus did not cope with the second problem mentioned above. Usually, one distinct time point was used to reconstruct the sources of the averaged IED (e.g. the peak or the 50\% slope of the peak of the IED) (Brodbeck et al. 2011) or, in case of ictal recordings, averaged discharges during ictal rhythms Assaf and Ebersole 1997; Ebersole 2000; Habib et al. 2015). In other ictal cases, epochs rather than single time points were reconstructed and the source with the highest amplitude over time and space was selected 75 (Koessler et al. 2010). In another approach, ESI depended on components of the decomposed EEG associated with seizure activity, with every component corresponding to a single topography to remove the potential non-stationarity of the signals (Jung et al. 2009, Yang et al. 2011). It cannot, however, be assured that the selected time sample or epoch contains only activity of the onset and so no propagated activity. Moreover, there is no direct evidence to assume that the brain activity in the SOZ is stronger than the propagated activity, because a single driving neuron could trigger a larger group of neurons resulting in an area of higher power elsewhere. 
The concept of functional brain connectivity can be used to investigate the epileptic network and its pathways. Functional brain connectivity models reveal how information flows are directed in the brain when applied on the reconstructed brain signals after ESI. Only a few studies tackling the two major problems stated above by combining ESI with functional connectivity analysis to localize the IZ/SOZ or study the epileptic networks have been performed up to date. Song et al. (2013) and Coito et al. (2015) investigated the epileptic network during IEDs in hd-EEG recordings. Song et al. (2013) applied ESI with either minimum norm or cortical surface Laplacian constraints and used spectral coherence for functional connectivity analysis to search for the possible engagement of pathological networks. They saw characteristic source coherence ${ }_{95}$ patterns before, during and after IEDs, but these patterns could not always be easily related to the EZ. Coito et al. (2015) performed source reconstruction using Local Auto-Regressive Averages (LAURA) and used Partial Directed Coherence (PDC) to assess the connectivity to investigate the fast-varying behavior of epileptic networks during interictal spikes and concluded that there are significantly different connectivity patterns in patients with left temporal lobe epilepsy, right temporal lobe epilepsy and healthy controls. These studies had as main goal to map the brain networks and to investigate their properties, rather than localizing the IZ or SOZ. Ding et al. (2007) and Lu et al. (2012) analyzed ictal epochs of $3 \mathrm{~s}$ by combining first principle vector (FINE) spatio-temporal ESI and directed transfer function (DTF) analysis to identify the ictal sources. While Ding et al. (2007) only used 32 electrodes, Lu et al. (2012) compared 32 and 76 electrodes and found more localizing results for the higher number of electrodes. Ding et al. (2007) estimated the SOZ within $15 \mathrm{~mm}$ of the presumed EZ in five patients and Lu et al. (2012) found the SOZ in 7 out of 10 patients within $10 \mathrm{~mm}$ of the RZ. Elshoff et al. (2013) analyzed ictal EEG segments (38-50 electrodes) of max. $10 \mathrm{~s}$ in the beginning and in the middle of seizures with Dynamic Imaging of Coherent Sources (DICS) to determine the SOZ. The source with the highest power was identified as SOZ. Afterwards, functional connectivity analysis based on (renormalized) partial directed coherence ((R)PDC) 


\section{Methods}

\subsection{Generation of simulated data}

Ictal EEG epochs of $3 \mathrm{~s}$ were constructed by forward projection of a simulated epileptic network in the brain. The details on the forward models used for this purpose, can be found in section 2.3.2. The epileptic network consisted of three nodes, of which the configuration can be seen in Fig. 1(a). The seizure originated in node 1 and propagated to node 3 via node 2 . In node 1 , the driver of the network, epileptic activity was mimicked by a sinusoid of decreasing frequency from $12 \mathrm{~Hz}$ at $t=0 \mathrm{~s}$ to $8 \mathrm{~Hz}$ at $t=3 \mathrm{~s}$ and its first two harmonics. Gaussian noise with $1 /$ f spectral behavior was added with a signal-to-noise ratio (SNR) of $5 \mathrm{~dB}$ to account for background brain activity. The seizure propagated to the second node with a delay of $20 \mathrm{~ms}$. Extra Gaussian 1/f noise is added with an SNR of $5 \mathrm{~dB}$. The resulting signal is delayed with $32 \mathrm{~ms}$ to node 3 and again 
Gaussian $1 / \mathrm{f}$ noise is added. The three signals were constructed with a sample (6) pre- and post-operative T1-weighted MRI of the patient was available. Five patients (2 male) with mean age of 37.6 years fulfilled all criteria and were in- 
cluded. Table 1 gives an overview of the patients' age and sex, clinical and MRI findings, the result of visual analysis of the scalp EEG, the performed resective surgery, and the outcome of the surgery. The local ethical committee approved the study and all patients gave written informed consent.

Long term hd-EEG was recorded for approximately 24 hours in each patient (EGI, Geodesic Sensor Net with 256 electrodes). From the 256 electrodes, the facial electrodes and the bottom line of the cap were removed due to major muscle artifacts, resulting in a setup of 204 electrodes. Electrode positions were estimated for every patient by manually fitting a template cap on the individual MRI. Also for the patient data, subsets of 200, 196, 192, 188, 184, 180, 176, 172, $168,164,128,64$ and 32 electrodes were created, with the configurations shown in Fig. 3. The sample frequency was either $250 \mathrm{~Hz}$ or $1000 \mathrm{~Hz}$. In the latter case, the EEG was downsampled offline to $250 \mathrm{~Hz}$ for consistency and to reduce computation time.

For every patient, an epoch of $3 \mathrm{~s}$ was selected that started at the seizure onset time marked by an EEG expert (MG).

\subsection{From EEG to $S O Z$}

An overview of the presented approach to get from the EEG to SOZ estimation is shown in Fig. 4. The data of patient 1 is used for illustration purpose. To summarize, we selected 3 seconds of ictal hd-EEG beginning at the marked seizure onset and reconstructed the sources generating the ictal brain activity with ESI. In the inverse solution, we selected local hotspots of higher activity. This resulted in 8 sources. As no constraints on the orientation of the sources were applied in the reconstruction, each of these 8 sources is represented by three time series, for the $\mathrm{x}, \mathrm{y}$ and $\mathrm{z}$ direction. We used singular value defomposition (SVD) to represent each source with one time series Golub and

Reinsch 1970). Using these time series, functional connectivity analysis was applied with the spectrum-weighted adaptive transfer function (swADTF) van Mierlo et al. 2013). Next, the swADTF values were summed to get the outdegree of every source as a measure for the total outgoing information flow 
from a source. The source with the highest outdegree, source 2 in the example (Fig. 4(g)), was selected as SOZ. This estimated SOZ was compared to the RZ of the patient and also to the source with highest power after ESI, i.e. source 3. In the following sections, we present every step of this method in detail.

\subsubsection{EEG preprocessing}

The patient data was common average referenced and band-pass filtered between 1 and $30 \mathrm{~Hz}$, to remove baseline drift and to reduce high frequency noise resulting from movement artifacts. An extra notch filter at $50 \mathrm{~Hz}$ was applied to filter out remaining power line noise. In patient 4 and 5, ICA was applied to remove remaining artifact (Makeig et al. 1996)..

\subsubsection{EEG source imaging}

To reconstruct the sources generating the ictal epochs, EEG source imaging (ESI) was done. For the forward model, patient-specific head models were constructed based on the finite difference method (FDM) (Hallez et al. 2005). Air, scalp, skull, cerebrospinal fluid (CSF), gray and white matter were segmented from the individual pre-operative T1-weighted MRI of the patient (resliced to voxels of $1 \times 1 \times 1 \mathrm{~mm}^{3}$ ) using the Statistical Parametric Mapping (SPM12) toolbox (http://www.fil.ion.ucl.ac.uk/spm). The segmented volumes were combined into a single head model with 6 tissue classes, and following conductivity values were assigned to the different tissues: $0.33 \mathrm{~S} / \mathrm{m}$ for gray matter, $0.14 \mathrm{~S} / \mathrm{m}$ for white matter, $1.79 \mathrm{~S} / \mathrm{m}$ for CSF (Baumann et al. 1997), $2250.33 / 25 \mathrm{~S} / \mathrm{m}=0.0132 \mathrm{~S} / \mathrm{m}$ for the skull and $0.33 \mathrm{~S} / \mathrm{m}$ for scalp (MontesRestrepo et al. 2014; Vorwerk et al. 2014), and $0 \mathrm{~S} / \mathrm{m}$ for air. The solution space was created based on the segmented gray matter. Solution points (SP) were uniformly distributed in the gray matter of the patient with a grid spacing of $4 \mathrm{~mm}$, which resulted in approximately $8000 \mathrm{SP}$ for every patient. These SPs formed the central nodes of the dipole model, so we ensured that at least 2 voxels of gray matter were left open between the SPs and the boundaries with other tissues in all directions, in order to keep the dipoles restricted to the gray 
matter.

An in-house implementation of the LOw Resolution Electromagnetic Tomog-

connections. As a consequence, the radius should be chosen in a range that keeps 
the number of selected sources computationally feasible and does not make the search area unnecessarily large, while not excluding possible network nodes (i.e. not excluding possibly relevant local maxima). We found good correspondence between the LORETA solution and the selected sources when a radius between 15 and $25 \mathrm{~mm}$ was chosen. We eventually used a radius of $20 \mathrm{~mm}$ as this provided overall best results and for which the number of selected sources varied between 4 and 13 for all patients during the analyzed ictal epoch.

Suppose that $K$ sources were selected. As LORETA does not impose constraints on dipole orientation, the activity in each source $k$ of the $K$ selected sources can be represented by a matrix $\mathbf{F}_{k} \in \mathbb{R}^{3 \times N}$ for $k=1 \ldots K$, with $N$ the number of time samples of the epoch. Each row of the matrix corresponds with an orthogonal spatial direction $(\mathrm{x}, \mathrm{y}, \mathrm{z})$. We used singular value decomposition

${ }_{275}$ (SVD) to represent each source by a single time series $\mathbf{s}_{k} \in \mathbb{R}^{N}$, associated with the largest singular value of the SVD (Golub and Reinsch 1970).

\subsubsection{Functional connectivity analysis}

Functional connectivity analysis was used to estimate which source is the most important, i.e. the driver behind the epileptic network. We used a Granger causality based measure to investigate the network, more specifically the spectrum-weighted adaptive directed transfer function (swADTF). The general concepts of this technique have been previously described by van Mierlo et al. (2013). First, the source signals $\mathbf{s}_{k}$ are modeled with a time-varying multivariate autoregressive (TVAR) model in which the signals are represented as a linear combination of their own past plus additional uncorrelated white noise. This can be mathematically described as:

$$
\mathbf{s}_{k}(t)=\sum_{p=1}^{P} \sum_{j=1}^{J} \mathbf{a}_{k j}(p, t) \mathbf{s}_{j}(t-p)+\mathbf{e}_{k}(t)
$$

in which $P$ is the model order, i.e. the number of past samples that are taken into account for the calculation of the current sample, $\mathbf{a}_{k j}(p, t)$ are the model coefficients, and $\mathbf{e}_{k}(t)$ is uncorrelated white noise at time $t$. In matrix represen- 
tation we get:

$$
\mathbf{S}(t)=\sum_{p=1}^{P} \mathbf{A}_{p}(t) \mathbf{S}(t-p)+\mathbf{E}(t)
$$

with $\mathbf{S}(t)=\left[\mathbf{s}_{1}(t) \mathbf{s}_{2}(t) \ldots \mathbf{s}_{K}(t)\right]^{T}$ the $K \times 1$ source matrix of the $K$ selected sources at time $t$, with $\mathbf{A}_{p}(t)$ the $K \times K$ coefficient matrix for delay $p$ at time $t$, modeling abrupt changes.

The time-varying transfer matrix $\mathbf{H}(f, t)$ of the model can be found after Fourier transformation and inversion of the coefficient matrix:

$$
\begin{aligned}
& \mathbf{A}(f, t)=-\sum_{p=0}^{P} \mathbf{A}_{p}(t) e^{-i 2 \pi \frac{f}{f_{s}} p} \\
& \mathbf{H}(f, t)=\mathbf{A}^{-1}(f, t)
\end{aligned}
$$

with $f_{s}$ the sample frequency and $\mathbf{A}_{p=0}(t)$ equal to the negative $K \times K$ identity matrix. The elements $\mathbf{H}_{i, j}(f, t)$ of the transfer matrix describe the information flow from signal $j$ to signal $i$ at frequency $f$ at time $t$. From the 
transfer matrix, the spectrum-weighted Adaptive Directed Transfer Function (swADTF) can be calculated to investigate the causal relation from source signal $\mathbf{s}_{j}$ to source signal $\mathbf{s}_{i}$ for a predefined frequency band at time $t$ :

$$
\operatorname{swADTF}_{i j}(t)=\frac{\sum_{f=f_{1}}^{f_{2}}\left|H_{i j}(f, t)\right|^{2} \sum_{k=1}^{K}\left|H_{j k}(f, t)\right|^{2}}{\sum_{l=1}^{K} \sum_{f^{\prime}=f_{1}}^{f_{2}}\left(\left|H_{i l}\left(f^{\prime}, t\right)\right|^{2} \sum_{o=1}^{K}\left|H_{l o}\left(f^{\prime}, t\right)\right|^{2}\right)}
$$

Due to normalization of the swADTF, the sum of incoming information flow into a source at each time point is equal to 1 :

$$
\sum_{j=1}^{J} \operatorname{swADTF}_{i j}(t)=1
$$

\subsubsection{SOZ localization}

The swADTF values were calculated for every source $\mathbf{s}_{j}$ to every other source $\mathbf{s}_{i}$ at every time point of the epoch in the frequency band $3-30 \mathrm{~Hz}$, as this band contained the fundamental seizure frequency noticed in the EEG. For every source $\mathbf{s}_{j}$, we defined the outdegree (OD) as the sum of the swADTF values to all other sources over time:

$$
\mathrm{OD}_{j}=\sum_{k=1}^{K} \sum_{t=1}^{T} \operatorname{swADTF}_{k j}(t)
$$

in which we defined $\operatorname{swADTF}_{j j}=0$. The outdegree is a measure for the total outgoing information flow from a source to all the other sources. The source with the highest outdegree was assumed to drive the epileptic network active during the seizure and was selected as SOZ.

\subsection{Evaluation of the simulated data}

With the forward model of every of the 5 patients, we simulated 200 ictal hd-EEG epochs of 3 seconds, resulting in a dataset of 1000 unique epochs. For all these epochs and all electrode setups, we tried to localize the driving patch of the epileptic network. The localization error $\left(\mathrm{LE}_{\mathrm{conn}}\right)$ was determined as the Euclidean distance between the border of the driving patch and the estimated SOZ, i.e. the source with the highest outdegree. If the selected SOZ was in the 
driving patch, the LE was $0 \mathrm{~mm}$. $\mathrm{LE}_{\mathrm{conn}}$ was then compared to the shortest distance between the driving patch and the source with the highest power $\left(\mathrm{LE}_{\mathrm{pow}}\right)$, to see whether connectivity analysis can provide extra information compared to ESI alone. Also the distance $\mathrm{LE}_{\min }$ between the driving patch and the closest source of all selected sources to the driving patch was calculated to provide a measure for the quality of the combination of ESI and source selection. It offers a lower bound on the error of both the source with the highest outdegree and the source with the highest power. When $\mathrm{LE}_{\text {conn } / \text { pow }}=\mathrm{LE}_{\min }$, the respective method achieves the best possible result, given the reconstructed sources. The different localization errors are illustrated in Fig. 5.

\subsection{Validation in patient data}

For all patients, we segmented the resected zone (RZ) from the post-operative MRI, which we coregistered to the solution space. We used the proposed approach to try to localize the SOZ for every patient and every electrode setup. Also for the patient data $\mathrm{LE}_{\mathrm{conn}}, \mathrm{LE}_{\text {pow }}$ and $\mathrm{LE}_{\min }$ were calculated, but now 325 with the RZ as a reference.

\section{Results}

\subsection{Simulated data}

\subsubsection{Overall results}

The results of the SOZ localization based on 1000 simulated ictal EEG epochs can be seen in Fig. 6. The data is represented in a boxplot, with the dot indicating the mean and the bar indicating the median of the errors. The data for 200, 192, 184, 176 and 168 electrodes are not shown, as they are very similar to their neighboring setups. From the figure, it is clear that connectivity analysis is better in localizing the epileptic driving patch than localization based on maximal power. More precisely, the localization error based on connectivity analysis was smaller than or equal to that based on power, $\mathrm{LE}_{\text {conn }} \leq \mathrm{LE}_{\text {pow }}$ in $85.5 \%$ of all the cases. $\mathrm{LE}_{\text {conn }}$ was strictly smaller than $\mathrm{LE}_{\text {pow }}$ in $58.5 \%$ 
and they were equal in $26.9 \%$ of all cases. Only in $14.5 \%$, power outperformed connectivity analysis.

When comparing the localization errors based on connectivity and power with the minimal error that could be achieved, we found that $\mathrm{LE}_{\mathrm{conn}}$ equaled $\mathrm{LE}_{\min }$ in $66.74 \%$ of the cases. This is in contrast with $\mathrm{LE}_{\text {pow }}$, which was equal to $\mathrm{LE}_{\min }$ in only $31.64 \%$.

\subsubsection{Influence of the number of electrodes}

345

From Fig. 6, it can be seen that the localization errors are not distributed normally. Therefore, we consider the median to be more useful than the mean for representing the data over the different electrode setups. An overview of the different medians can be found in Table 2 The median of the minimal localization error $\mathrm{LE}_{\min }$ was smaller than $10 \mathrm{~mm}$ for all setups except for 32 electrodes, for which it was $12 \mathrm{~mm}$. The upper quartile stayed below $20 \mathrm{~mm}$. For all electrode setups, it was possible to find a source very close to the origin of the simulated seizure. The median of the localization error based on connectivity analysis $\mathrm{LE}_{\text {conn }}$ was smaller than $15 \mathrm{~mm}$ in all setups, except for 32 electrodes, for which it was $20.78 \mathrm{~mm}$. We notice an increase in both $\mathrm{LE}_{\min }$ and $\mathrm{LE}_{\mathrm{conn}}$ when we lowered the number of electrodes, especially in the upper quartile of $\mathrm{LE}_{\text {conn }}$. Between 32 and 204 electrodes, the median of $\mathrm{LE}_{\min }$ got $5.07 \mathrm{~mm}$ larger and the median of $\mathrm{LE}_{\mathrm{conn}} 8.78 \mathrm{~mm}$, which represented in both cases an increase of approximately $72 \%$. In contrast, the median of the localization error based on power was much larger and varied between 40.10 and $44.72 \mathrm{~mm}$ over all electrode setups, which reflected a fluctuation of maximal $11.5 \%$.

\subsection{Patient data}

\subsubsection{Overall results}

In Fig. 7, an overview of the localization errors (LEs) for all patients and all electrode setups can be found. A localization error of e.g. $50 \mathrm{~mm}$ is considered to be as unfavorable as a localization error of $80 \mathrm{~mm}$, therefore we used 
different intervals to characterize the errors: $\mathrm{LE}=0 \mathrm{~mm}, \mathrm{LE} \in] 0,10] \mathrm{mm}$, $\mathrm{LE} \in] 10,25] \mathrm{mm}, \mathrm{LE} \in] 25,45] \mathrm{mm}$, and $\mathrm{LE}>45 \mathrm{~mm}$.

From the figure, we can see that $\mathrm{LE}_{\mathrm{conn}}$ was equal to or smaller than $\mathrm{LE}_{\mathrm{pow}}$ in $91.4 \%$ of the cases, meaning that in these cases our presented method performed

as well as or better than localization based on power. $\mathrm{LE}_{\mathrm{conn}}$ was strictly smaller than $\mathrm{LE}_{\text {pow }}$ in $57.1 \%$ of the cases. In $34.3 \%, \mathrm{LE}_{\mathrm{conn}}=\mathrm{LE}_{\text {pow }}$ and, in $8.6 \%$ of the cases, power outperformed connectivity analysis, $\mathrm{LE}_{\mathrm{conn}}>\mathrm{LE}_{\text {pow }}$. These $8.6 \%$ represent 6 cases that are mainly located in the low-density setups (3 for 32 electrodes and 1 for 64 electrodes, and two outliers for p1 for 184 and 192 electrodes).

For all setups, ESI and source selection were able to find a source inside the RZ for 4 patients, and within $10 \mathrm{~mm}$ of the border of the RZ or within the resection for the other patient. Connectivity analysis was able to find this optimal solution in $88.6 \%$ of the cases, whereas $\mathrm{LE}_{\text {pow }}=\mathrm{LE}_{\min }$ in $38.6 \%$ of the cases.

\subsubsection{Influence of the number of electrodes}

Fig. 8 shows the result of the connectivity analysis and the source with the highest power compared to the RZ for all patients for 204 electrodes. For the 204 electrodes setup, we were able to estimate the SOZ inside the $\mathrm{RZ}\left(\mathrm{LE}_{\mathrm{conn}}=0 \mathrm{~mm}\right.$ in $\mathrm{p} 1, \mathrm{p} 2, \mathrm{p} 4, \mathrm{p} 5)$ or within $10 \mathrm{~mm}$ of the border $\left(\mathrm{LE}_{\mathrm{conn}}<10 \mathrm{~mm}\right.$ in $\left.\mathrm{p} 3\right)$ of the RZ. In contrast, localization based on power was only able to estimate the SOZ inside the RZ in one patient and within $10 \mathrm{~mm}$ of the border of the RZ in one other patient. In these cases, localization based on power and connectivity found the same source. For the three other patients, the localization error LE $\mathrm{L}_{\text {pow }}$ was larger than 25 (1/3 patients) or $45 \mathrm{~mm}$ (2/3 patients). The lateralization was, however, correct. When comparing to the minimal localization error, connectivity analysis achieved the best possible result after ESI and source selection in every patient for 204 electrodes. This is also shown in Fig. 9, which displays in how many patients the minimal localization error was found, for every electrode setup and for both methods. On the contrary, localization based on power was 
only able to select the optimal source in 2 patients. The same results applied when gradually lowering the number of electrodes to 128 , with the exception of three cases: for 192 and 184 electrodes, localization based on connectivity analysis does not find the optimal source in p1. For 128 electrodes, power localized the optimal source only in 1 out of 5 patients. For the high-density setups we can say that the presented approach outperformed localization based on power consistently.

For 64 electrodes, the performance of the presented approach decreased. The SOZ was estimated inside the RZ (2/5 patients) or within $10 \mathrm{~mm}$ of the border of the RZ (1/5 patients) in only 3 patients instead of 5 . Yet, this result is better than localization based on power, for which the optimal source is only found in 2 patients. Only for one patient $\mathrm{LE}_{\text {pow }}$ was smaller than $10 \mathrm{~mm}$. This is also reflected in Fig. 9: $\mathrm{LE}_{\text {conn }}=\mathrm{LE}_{\min }$ in 3 patients and $\mathrm{LE}_{\mathrm{pow}}=\mathrm{LE}_{\min }$ in one patient.

410

For the low-density setup of 32 electrodes, the $\mathrm{SOZ}$ was estimated inside the $\mathrm{RZ}$ in only one patient. The source with the highest power, was however inside the RZ in 2 out of 5 patients.

\section{Discussion}

In this paper, we proposed an approach that combines ESI and functional connectivity analysis to localize the SOZ from non-invasive EEG in patients with refractory epilepsy. We look at the connectivity instead of the power of the neuronal activity during an epileptic seizure. The presented method does not require patient-dependent parameters, which makes it suitable for use in clinical practice. We compared the localization obtained from connectivity measures with the maximal power of the electrical activity at the onset of an epileptic seizure.

We validated our method using simulated ictal EEG epochs and found that localization based on connectivity analysis had a significantly and consistently better yield than localization based on maximal power, for every electrode setup. 

which is in agreement with literature (Michel and Murray 2012). As a result, the perfomance of connectivity analysis also increased for high-density setups. The influence of the amount of electrodes was much smaller when localization was based on maximal power, but the median localization error was unacceptably high for all setups.

Next to simulations, we validated the method in five patients. For almost all high-density setups with 128 electrodes or more, we found the best possible result with the presented method: in four out of five patients the connectivity analysis selected the best possible source to localize the SOZ in every setup. source in all but two setups. The source with the highest power coincided with this optimal source in only two out of five patients. These results are better than what we found with the simulations, but this can be accounted for by the resected zones of the patients being larger than the patches of the simulated network. Next, we found equally good or better results with the connectivity method in $91.4 \%$ of the cases compared to selecting the source with the highest power. A possible explanation for this could be that there is some remaining artifact in the selected epochs, and that connectivity analysis is more robust to artifacts and noise in the EEG than the power metric. A solution would be to limit the power analysis to a patient-specific seizure frequency range, to filter out the artifact as much as possible. This is done by Elshoff et al. (2013), where the frequency range could also change over the course of the seizure. However, given that a patient-specific seizure range could make the method subjective and less suitable to be directly used in a clinical setting, we opted not to do this and performed wide-spectrum analysis.

In the range of 204 down to 128 electrodes, our method generally estimated the localization of the SOZ inside $(4 / 5$ patients $)$ or very close $(<10 \mathrm{~mm})$ to the boundary of the RZ (1/5 patients). When lowering the number of electrodes down to 64 , the performance of the method dropped, but it was still capable of 455 localizing the SOZ inside (2/5 patients) or very close to the border of the RZ 
(1/5 patients). For the low-density setup with 32 electrodes, we experienced an extra drop in performance. When using only 32 electrodes, there was correct localization in only one of the patients. Setups with more electrodes are thus preferred in the current approach. We assume that the performance goes down with the number of electrodes due to suboptimal estimation of the time series per source (however the goodness-of-fit did not drop significantly) and/or SVD not being able to represent the three orthogonal time series as one time series. As a consequence, these errors propagate in the connectivity analysis and the correct source cannot be selected. Nevertheless, Ding et al. (2007) used only 31 electrodes and they were able to localize the SOZ within $15 \mathrm{~mm}$ of the presumed EZ.

However, four out of the five analyzed patients showed clear large lesions on MRI that were presumed to be epileptogenic and it remains to be investigated how this influenced the results, as only one patient in this study had a small

lesion. Furthermore, it is not addressed whether the patients had surgery, and if so, whether surgery was successful. There were also no intracranial EEG recordings to validate the results. Lu et al. (2012) performed a similar study with 76 electrodes, and they were able to localize the SOZ within $10 \mathrm{~mm}$ of the border of the RZ in 7 out of 10 patients. This result is comparable with our study for the 64 electrode setup.

To improve results for lower-density setups, some suggestions can be made. A possible improvement could be to use patient-specific electrode locations in the forward model. The benefit of this has been investigated and could improve the estimation of the time series corresponding to each selected source Van Hoey 480 et al. 2000: Wang and Gotman 2001; Dalal et al. 2014). We chose LORETA, as inverse solution method as it is a simple, clear and easily controllable technique fit for the reconstruction of non-stationary signals that was ready at hand in our group. It proved itself suited for the proposed framework, however, the influence of other, more advanced inverse techniques could be investigated, such as the multiple sparse volumetric priors (MSVP) algorithm (Strobbe et al. 2014), the FINE algorithm (Xu et al. 2004, Ding and He 2006; Ding et al. 2007), 
dynamic imaging of coherent sources (DICS) (Groß et al. 2001, 2002), or the Maximum Entropy on the Mean (MEM) approach (Clarke and Janday 1989. Grova et al. 2006). In our analysis, the inverse solution is estimated for every time sample separately, but we could take into account other time samples to improve ESI. Other connectivity measures and/or graph theory measures could be considered. Some connectivity measures related to the swADTF were tested, the integrated ADTF (iADTF) and the full-frequency ADTF (ffADTF) (van Mierlo et al. 2011), both resulting in worse results. Another interesting approach for SOZ localization is to first decompose the ictal data to isolate seizure components, e.g. with ICA, and then integrate ESI with a recombination approach. This is done by Yang et al. (2011), where this dynamic source imaging technique identified ictal activity in good correlation with iEEG and surgical outcomes. It remains to be investigated how functional connectivity analysis can possibly enhance this method.

ESI resulted in all cases in a source close to the resection, reflected in an overall low $\mathrm{LE}_{\text {min }}$. The selection of this optimal source was significantly better using connectivity analysis compared to selecting the source with the highest power, especially in high-density setups.

When applying functional connectivity analysis on non-invasive recordings, the volume conduction problem is a well-known phenomenon. Due to the large distance between the sources and the electrodes and the different tissues the sources have to propagate through, a single source will be seen at several electrodes. We addressed this problem by demixing the sources, i.e. with ESI, but this technique does not mitigate the effects of volume conduction completely and spurious connections can still possibly exist (Schoffelen and Gross 2009). There is no technique to completely alter the mixing problem, but it would be interesting to compare the current framework with other techniques, e.g. the imaginary part of the coherency (Nolte et al. 2004), designed to undo the volume conduction problem in combination with functional connectivity analysis. A clear review of the volume conduction problem in functional connectivity analysis and different strategies and techniques to solve it, is given by Bastos 
and Schoffelen (2015).

Considering the patient data, an important remark to make is that the

\section{Conclusion}

We developed a method based on ESI and functional connectivity analysis to localize the seizure onset zone in a non-invasive, objective way that can potentially be used in a clinical setting. The approach consistently outperformed 
diverse patient group is warranted. We conclude that our presented approach and in general ESI combined with functional connectivity analysis can serve as a useful tool for SOZ localization in the presurgical evaluation of epilepsy. der the Marie Sklodowska-Curie Grant No. 660230 (P. van Mierlo).

\section{References}

Akaike H (1974) A new look at the statistical model identification. IEEE Trans Autom Control 19(6):716-723

Arnold M, Milner X, Witte H, Bauer R, Braun C (1998) Adaptive AR modeling of nonstationary time series by means of Kalman filtering. IEEE Trans Biomed Eng 45(5):553-562

Assaf BA, Ebersole JS (1997) Continuous source imaging of scalp ictal rhythms in temporal lobe epilepsy. Epilepsia 38(10):1114-1123

Astolfi L, Cincotti F, Mattia D, de Vico Fallani F, Tocci A, Colosimo A, Salinari S, Marciani MG, Hesse W, Witte H, et al. (2008) Tracking the timevarying cortical connectivity patterns by adaptive multivariate estimators. IEEE Trans Biomed Eng 55(3):902-913

Bastos AM, Schoffelen JM (2015) A tutorial review of functional connectivity analysis methods and their interpretational pitfalls. Frontiers in systems neuroscience 9 
Baumann SB, Wozny DR, Kelly SK, Meno FM (1997) The electrical conductivity of human cerebrospinal fluid at body temperature. IEEE Trans Biomed Eng 44(3):220-223

Boon P, D'Havé M, Vanrumste B, Van Hoey G, Vonck K, Van Walleghem P, Caemaert J, Achten E, De Reuck J (2002) Ictal source localization in presurgical patients with refractory epilepsy. J Clin Neurophysiol 19(5):461468

Brodbeck V, Spinelli L, Lascano AM, Wissmeier M, Vargas MI, Vulliémoz S, Pollo C, Schaller K, Michel CM, Seeck M (2011) Electroencephalographic source imaging: a prospective study of 152 operated epileptic patients. Brain 134(10):2887-2897

Carrette E, Vonck K, Boon P (2011) The management of pharmacologically refractory epilepsy. Int J of Clinical Reviews 1(02)

Clarke C, Janday B (1989) The solution of the biomagnetic inverse problem by maximum statistical entropy. Inverse Probl 5(4):483

Coito A, Plomp G, Genetti M, Abela E, Wiest R, Seeck M, Michel CM, Vulliémoz S (2015) Dynamic directed interictal connectivity in left and right temporal lobe epilepsy. Epilepsia 56(2):207-217

Dalal SS, Rampp S, Willomitzer F, Ettl S (2014) Consequences of EEG electrode position error on ultimate beamformer source reconstruction performance. Front Neurosci 8

de Tisi J, Bell GS, Peacock JL, McEvoy AW, Harkness WF, Sander JW, Duncan JS (2011) The long-term outcome of adult epilepsy surgery, patterns of seizure remission, and relapse: a cohort study. The Lancet 378(9800):1388-1395

Ding L, He B (2006) Spatio-temporal EEG source localization using a threedimensional subspace fine approach in a realistic geometry inhomogeneous head model. IEEE Trans Biomed Eng 53(9):1732-1739 
Ding L, Worrell GA, Lagerlund TD, He B (2007) Ictal source analysis: localization and imaging of causal interactions in humans. Neuroimage 34(2):575-586

Ebersole JS (2000) Noninvasive localization of epileptogenic foci by EEG source modeling. Epilepsia 41(s3):S24-S33

Elshoff L, Muthuraman M, Anwar AR, Deuschl G, Stephani U, Raethjen J, Siniatchkin M (2013) Dynamic imaging of coherent sources reveals different network connectivity underlying the generation and perpetuation of epileptic seizures. PloS one 8(10):e78422

Golub GH, Reinsch C (1970) Singular value decomposition and least squares solutions. Numer Math 14(5):403-420

Groß J, Kujala J, Hämäläinen M, Timmermann L, Schnitzler A, Salmelin R (2001) Dynamic imaging of coherent sources: studying neural interactions in the human brain. Proc Natl Acad Sci 98(2):694-699

Groß J, Timmermann L, Kujala J, Dirks M, Schmitz F, Salmelin R, Schnitzler A (2002) The neural basis of intermittent motor control in humans. Proc Natl Acad Sci 99(4):2299-2302

Grova C, Daunizeau J, Lina JM, Bénar CG, Benali H, Gotman J (2006) Evaluation of EEG localization methods using realistic simulations of interictal spikes. Neuroimage 29(3):734-753

Haalman I, Vaadia E (1997) Dynamics of neuronal interactions: relation to behavior, firing rates, and distance between neurons. Hum Brain Mapp 5(4):249253

Habib MA, Ibrahim F, Mohktar MS, Kamaruzzaman SB, Rahmat K, Lim KS (2015) Ictal EEG source imaging for presurgical evaluation of refractory focal epilepsy. World Neurosurg

Hallez H, Vanrumste B, Van Hese P, D'Asseler Y, Lemahieu I, Van de Walle R (2005) A finite difference method with reciprocity used to incorporate 
anisotropy in electroencephalogram dipole source localization. Phys Med Biol $50(16): 3787$

Jayakar P, Duchowny M, Resnick TJ, Alvarez LA (1991) Localization of seizure foci: pitfalls and caveats. J Clin Neurophysiol 8(4):414-431

Jung KY, Kang JK, Kim JH, Im CH, Kim KH, Jung HK (2009) Spatiotemporospectral characteristics of scalp ictal EEG in mesial temporal lobe epilepsy with hippocampal sclerosis. Brain Res 1287:206-219

Koessler L, Benar C, Maillard L, Badier JM, Vignal JP, Bartolomei F, Chauvel P, Gavaret M (2010) Source localization of ictal epileptic activity investigated by high resolution EEG and validated by SEEG. Neuroimage 51(2):642-653

Lantz G, de Peralta RG, Spinelli L, Seeck M, Michel CM (2003) Epileptic source localization with high density EEG: how many electrodes are needed? Clin Neurophysiol 114(1):63-69

Lantz G, Michel CM, Seeck M, Blanke O, Landis T, Rosén I (1999) Frequency domain EEG source localization of ictal epileptiform activity in patients with partial complex epilepsy of temporal lobe origin. Clin Neurophysiol 110(1):176-184

Lu Y, Yang L, Worrell GA, He B (2012) Seizure source imaging by means of fine spatio-temporal dipole localization and directed transfer function in partial epilepsy patients. Clin Neurophysiol 123(7):1275-1283

Makeig S, Bell AJ, Jung TP, Sejnowski TJ, et al. (1996) Independent component analysis of electroencephalographic data. Adv Neur In 145-151

Mégevand P, Spinelli L, Genetti M, Brodbeck V, Momjian S, Schaller K, Michel CM, Vulliémoz S, Seeck M (2014) Electric source imaging of interictal activity accurately localises the seizure onset zone. J Neurol Neurosur Ps 85(1):38-43

Michel CM, Murray MM (2012) Towards the utilization of EEG as a brain imaging tool. Neuroimage 61(2):371-385 
Montes-Restrepo V, van Mierlo P, Strobbe G, Staelens S, Vandenberghe S, Hallez H (2014) Influence of skull modeling approaches on EEG source localization. Brain Topogr 27(1):95-111

Nolte G, Bai O, Wheaton L, Mari Z, Vorbach S, Hallett M (2004) Identifying true brain interaction from eeg data using the imaginary part of coherency. Clin Neurophysiol 115(10):2292-2307

Pascual-Marqui RD, Michel CM, Lehmann D (1994) Low resolution electromagnetic tomography: a new method for localizing electrical activity in the brain. Int J Psychophysiol 18(1):49-65

Plummer C, Harvey AS, Cook M (2008) EEG source localization in focal epilepsy: where are we now? Epilepsia 49(2):201-218

Rémi J, Vollmar C, de Marinis A, Heinlin J, Peraud A, Noachtar S (2011) Congruence and discrepancy of interictal and ictal EEG with MRI lesions in focal epilepsies. Neurology 77(14):1383-1390

Richardson MP (2012) Large scale brain models of epilepsy: dynamics meets connectomics. J Neurol Neurosur Ps 83(12):1238-1248

Rosenow F, Lüders H (2001) Presurgical evaluation of epilepsy. Brain 124(9):1683-1700

Schlögl A, Roberts S, Pfurtscheller G (2000) A criterion for adaptive autoregressive models. In P Ann Int IEEE EMBS, 1581-1582

Schoffelen JM, Gross J (2009) Source connectivity analysis with meg and eeg. Hum Brain Map 30(6):1857-1865

Smith S (2005) EEG in the diagnosis, classification, and management of patients with epilepsy. J Neurol Neurosur Ps 76(suppl 2):ii2-ii7

Song J, Tucker DM, Gilbert T, Hou J, Mattson C, Luu P, Holmes MD (2013) Methods for examining electrophysiological coherence in epileptic networks. Front Neurol 4 
Spencer SS (2002) Neural networks in human epilepsy: evidence of and implications for treatment. Epilepsia 43(3):219-227

Sprengers M, Vonck K, Carrette E, Marson AG, Boon P (2014) Deep brain and cortical stimulation for epilepsy. Cochrane Database Syst Rev 6

Strobbe G, Carrette E, López JD, Van Roost D, Meurs A, Vonck K, Boon P, Vandenberghe S, van Mierlo P (2016) Electrical source imaging of interictal spikes using multiple sparse volumetric priors for presurgical epileptogenic focus localization. Neuroimage Clin

Strobbe G, van Mierlo P, De Vos M, Mijović B, Hallez H, Van Huffel S, López JD, Vandenberghe S (2014) Multiple sparse volumetric priors for distributed EEG source reconstruction. Neuroimage 100:715-724

Téllez-Zenteno JF, Dhar R, Wiebe S (2005) Long-term seizure outcomes following epilepsy surgery: a systematic review and meta-analysis. Brain 128(5):1188-1198

Van Hoey G, Vanrumste B, D'Havé M, Van de Walle R, Lemahieu I, Boon P (2000) Influence of measurement noise and electrode mislocalisation on EEG dipole-source localisation. Med Biol Eng Comput 38(3):287-296

van Mierlo P, Carrette E, Hallez H, Raedt R, Meurs A, Vandenberghe S, Roost D, Boon P, Staelens S, Vonck K (2013) Ictal-onset localization through connectivity analysis of intracranial EEG signals in patients with refractory epilepsy. Epilepsia 54(8):1409-1418

van Mierlo P, Carrette E, Hallez H, Vonck K, Van Roost D, Boon P, Staelens S (2011) Accurate epileptogenic focus localization through time-variant functional connectivity analysis of intracranial electroencephalographic signals. Neuroimage 56(3):1122-1133

Vorwerk J, Cho JH, Rampp S, Hamer H, Knösche TR, Wolters CH (2014) A guideline for head volume conductor modeling in eeg and meg. Neuroimage 100:590-607 
Wang Y, Gotman J (2001) The influence of electrode location errors on EEG dipole source localization with a realistic head model. Clin Neurophysiol 112(9):1777-1780

Wennberg R, Cheyne D (2014) EEG source imaging of anterior temporal lobe spikes: validity and reliability. Clin Neurophysiol 125(5):886-902

Wennberg R, Valiante T, Cheyne D (2011) EEG and MEG in mesial temporal lobe epilepsy: where do the spikes really come from? Clin Neurophysiol 122(7):1295-1313

Xu XL, Xu B, He B (2004) An alternative subspace approach to EEG dipole source localization. Phys Med Biol 49(2):327

Yang L, Wilke C, Brinkmann B, Worrell GA, He B (2011) Dynamic imaging of ictal oscillations using non-invasive high-resolution eeg. Neuroimage 56(4):1908-1917 
Table 1 Overview of patient details. $\mathrm{L}=$ left, $\mathrm{R}=$ right, $\mathrm{M}=$ male, $\mathrm{F}=$ female, $\mathrm{TPO}=$ temporo - parieto - occipital

\begin{tabular}{|c|c|c|c|c|c|}
\hline & p1 & p2 & p3 & $\mathrm{p} 4$ & p5 \\
\hline Age (y) & 36 & 42 & 36 & 29 & 45 \\
\hline Sex & $\mathrm{F}$ & $\mathrm{F}$ & $\mathrm{M}$ & $\mathrm{F}$ & M \\
\hline Cinical findings & complex partial seizures & complex partial seizures & $\begin{array}{c}\text { epigastric aura, } \\
\text { secondary generalisation }\end{array}$ & $\begin{array}{c}\text { aura of deja vu, } \\
\text { change of taste, } \\
\text { non-lateralized } \\
\text { impairment of vision }\end{array}$ & $\begin{array}{l}\text { complex partial seizures, } \\
\text { general tonic clonic seizures }\end{array}$ \\
\hline MRI findings & $\mathrm{R}$ hippocampal sclerosis & $\mathrm{R}$ hippocampal sclerosis & $\mathrm{R}$ hippocampal slcerosis & $\begin{array}{l}\mathrm{R} \text { cortical dysplasia in } \\
\text { amygdala and } \\
\text { paraventricular nucleus }\end{array}$ & L TPO Gangliocytoma \\
\hline $\begin{array}{r}\text { Visual analysis of } \\
\text { interictal scalp EEG }\end{array}$ & $\mathrm{T} 2, \mathrm{~F} 8$ & TP10, T8 & $\mathrm{T} 2, \mathrm{~T} 8, \mathrm{P} 8, \mathrm{Tp} 10$ & $\mathrm{~T} 2, \mathrm{~T} 8$ & F3, P7, CP3, P5 \\
\hline Surgery & $\begin{array}{l}\mathrm{R} \text { polectomy and } \\
\text { amygdalohippo- } \\
\text { campectomy }\end{array}$ & $\begin{array}{l}\mathrm{R} \text { temporal } \\
\text { anterior lobectomy }\end{array}$ & $\begin{array}{l}\mathrm{R} \text { amygdalohippo- } \\
\text { campectomy }\end{array}$ & $\begin{array}{l}\mathrm{R} \text { polectomy and } \\
\text { temporal anterior lobectomy }\end{array}$ & L occipital lobectomy \\
\hline Engel Class & I & I & I & I & III \\
\hline
\end{tabular}


Table 2 Overview of the medians of $\mathrm{LE}_{\mathrm{conn}}, \mathrm{LE}_{\mathrm{min}}$ and $\mathrm{LE}_{\mathrm{pow}}$ for the different setups obtained after evaluation of 1000 simulated ictal EEG epochs

\begin{tabular}{|c|c|c|c|c|c|c|c|c|c|}
\hline$(\mathrm{mm})$ & 204 & 196 & 188 & 180 & 172 & 164 & 128 & 64 & 32 \\
\hline median $\mathbf{L} \mathbf{E}_{\text {conn }}$ & 12.00 & 12.00 & 12.00 & 12.65 & 12.65 & 12.65 & 13.27 & 14.97 & 20.78 \\
\hline median $L E_{\min }$ & 6.93 & 6.93 & 6.93 & 8.00 & 8.00 & 8.00 & 8.94 & 9.37 & 12.00 \\
\hline median $\mathbf{L E}_{\text {pow }}$ & 44.72 & 44.72 & 42.99 & 42.99 & 44.00 & 42.61 & 41.95 & 40.99 & 40.10 \\
\hline
\end{tabular}

Figures 


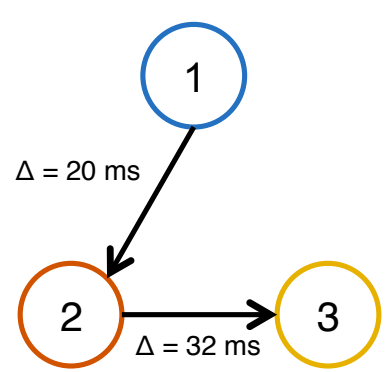

(a)

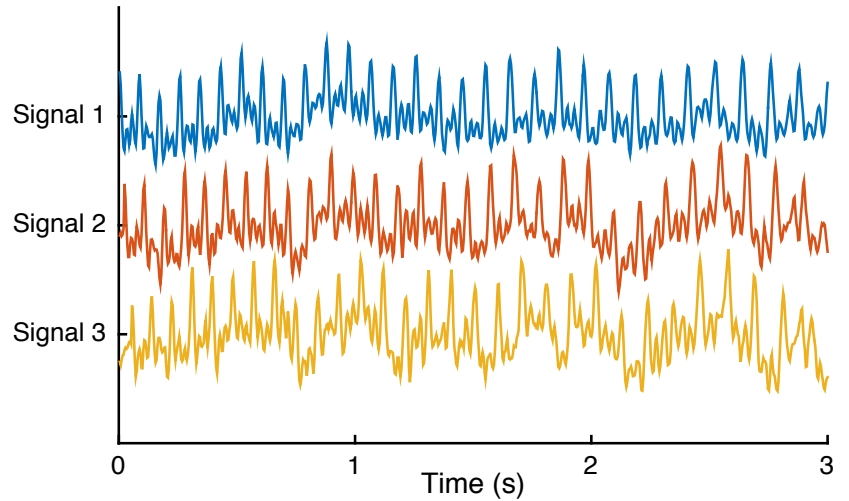

(b)

Figure 1 (a) Configuration of the simulated epileptic network. Source 1 is the overall driver.

(b) An example of the signals that mimick epileptic activity corresponding to the three nodes of the network

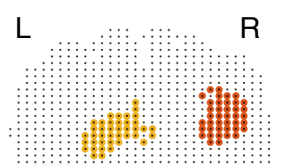

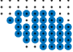

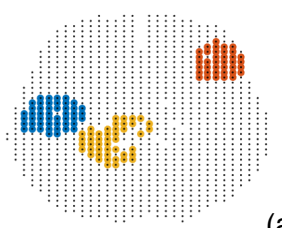

(a)

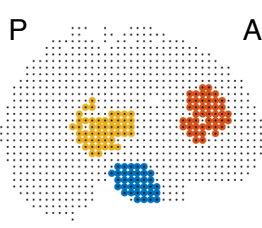

- patch node 1

- patch node 2

- patch node 3
A $\quad$ FP1-F7

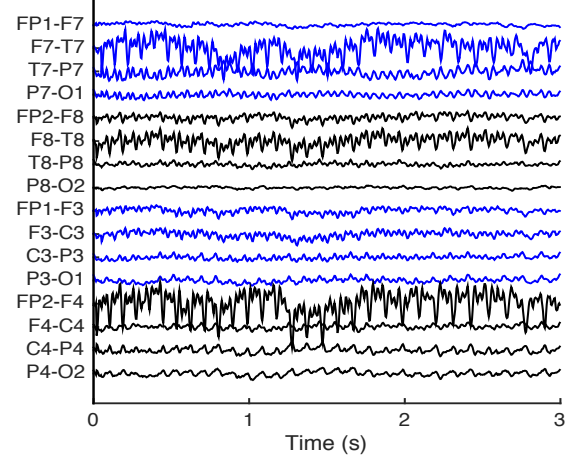

(b)

Figure 2 (a) Example of three randomly located epileptic patches in the brain, corresponding to the nodes of the network. (b) Montage of the resulting EEG after projection of the epileptic brain activity to sensor space 


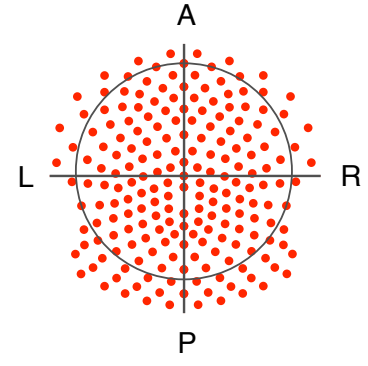

(a) 204

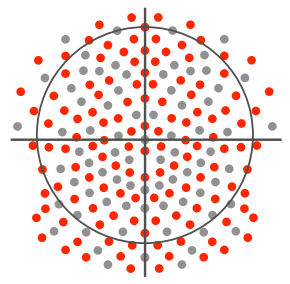

(d) 128

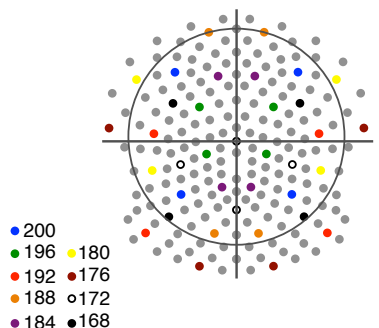

(b) $200-168$

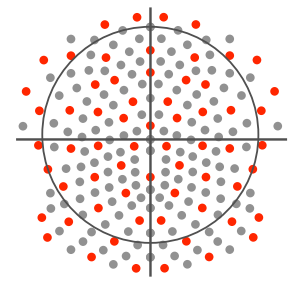

(e) 64

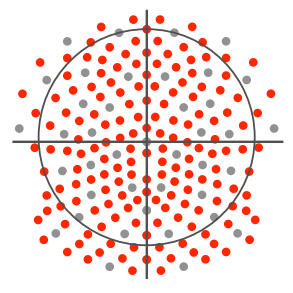

(c) 164

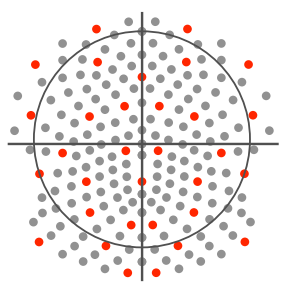

(f) 32

Figure 3 The different used electrode setups: (a) original 204 electrode setup, (b) the electrodes that were subsequently removed to obtain setups of 200-168 electrodes, (c) subset with 164 electrodes (red), (d) 128 electrodes, (e) 64 electrodes and (f) 32 electrodes. $\mathrm{L}=$ left, $\mathrm{R}=$ right, $\mathrm{A}=$ anterior, $\mathrm{P}=$ posterior 
(a) Ictal EEG epoch

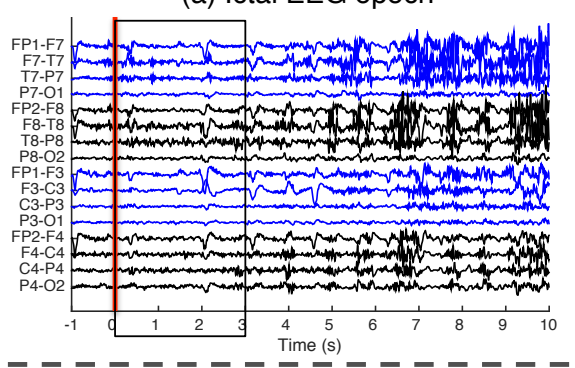

(c) Source selection

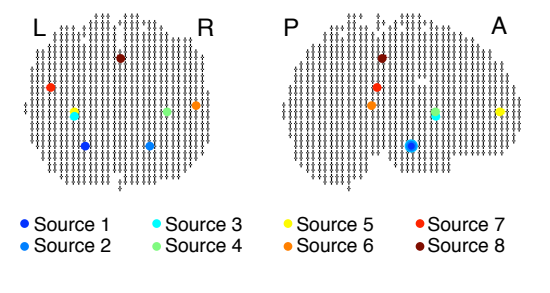

(e) SVD

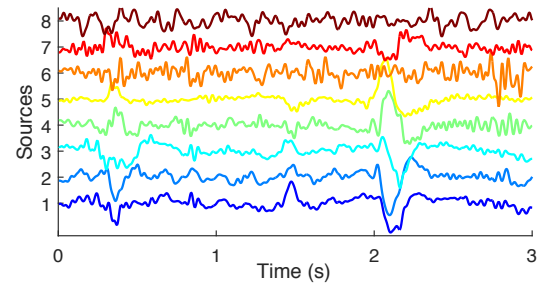

$---------\frac{\text { Time }(\mathrm{s})}{\text { (g) Outdegree }}$
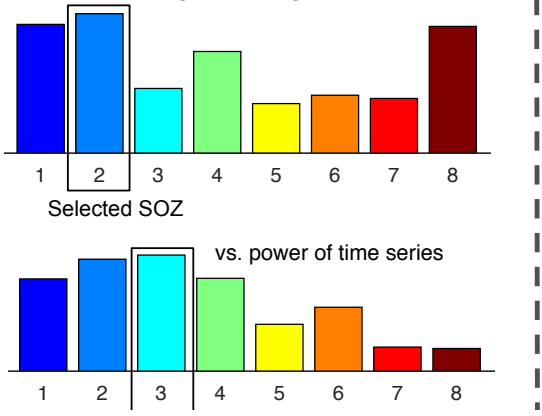

(b) ESI

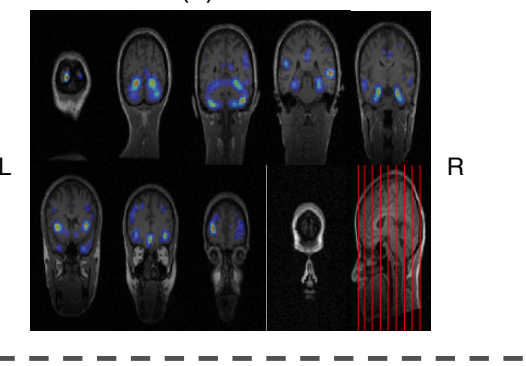

(d) corresponding time series

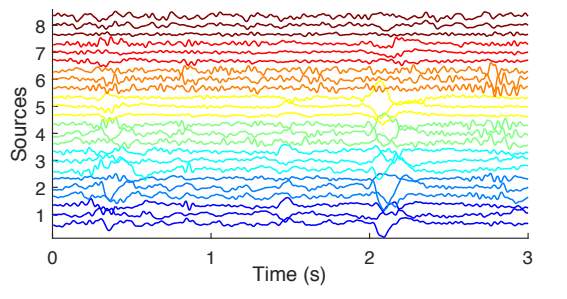

(f) SWADTF
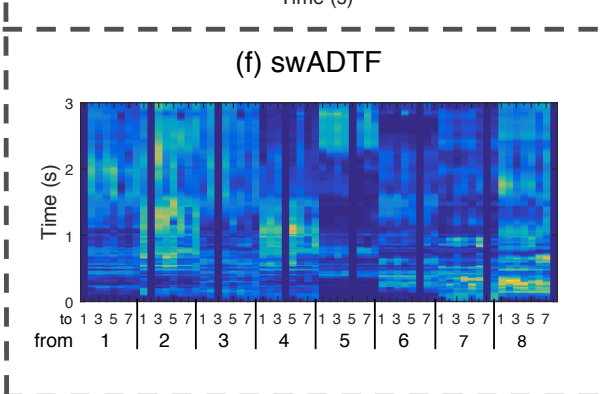

(h) SOZ

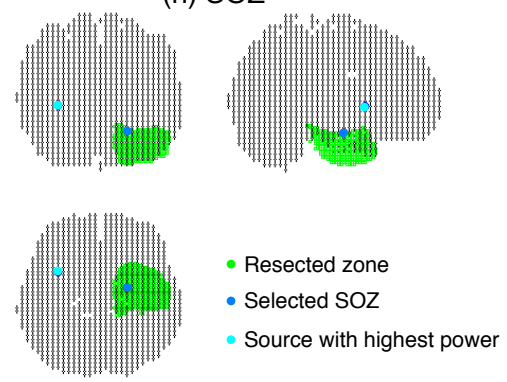

Figure 4 Analysis of p1. (a) Selection and preprocessing of an ictal hd-epoch. (b) Result of EEG source imaging, (c) Source selection shown in solution space and (d) time series for $\mathrm{x}, \mathrm{y}$ and $\mathrm{z}$ direction for these sources. (e) SVD to represent each source with one time series. (f) swADTF values over time (summed in the $3-30 \mathrm{~Hz}$ frequency band) for every source to every other source. (g) Summation of the swADTF values leads to the outdegree. (h) The source with the highest outdegree is selected as SOZ. The location of this source is compared with the location of the source with the highest power and with the segmented resected zone. The presented method finds the SOZ in the RZ, while the source with the highest power is not located inside the RZ 


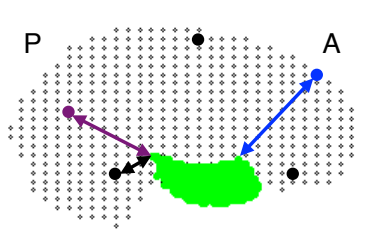

(a)

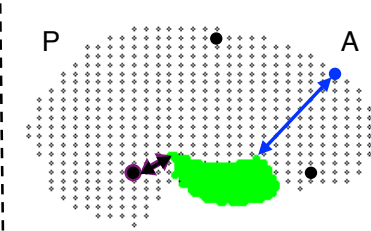

(b)

- Source with highest outdegree $\longleftrightarrow \mathrm{LE}_{\mathrm{conn}}$

- Source with highest power $\leftrightarrow \mathrm{LE}_{\text {pow }}$-driving patch/RZ

- Other selected sources

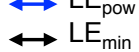

Figure 5 Illustration of the different localization errors. $\mathrm{LE}_{\mathrm{conn}}=$ the Euclidean distance between the source with the highest outdegree and the driving patch in the simulated data or the $\mathrm{RZ}$ in the patient data, $\mathrm{LE}_{\text {pow }}=$ the Euclidean distance between the source with the highest power and the driving patch/RZ, and $\mathrm{LE}_{\min }=$ the Euclidean distance between the source closest to the driving patch/RZ and the driving patch/RZ. (a) In this case $\mathrm{LE}_{\text {pow }}>\mathrm{LE}_{\mathrm{conn}}>\mathrm{LE}_{\text {min }}$. (b) The localization errors can be equal to each other. In this case $\mathrm{LE}_{\mathrm{conn}}=\mathrm{LE}_{\min }$, meaning that our method selected the best possible source to estimate the SOZ. A = anterior, $\mathrm{P}=$ posterior

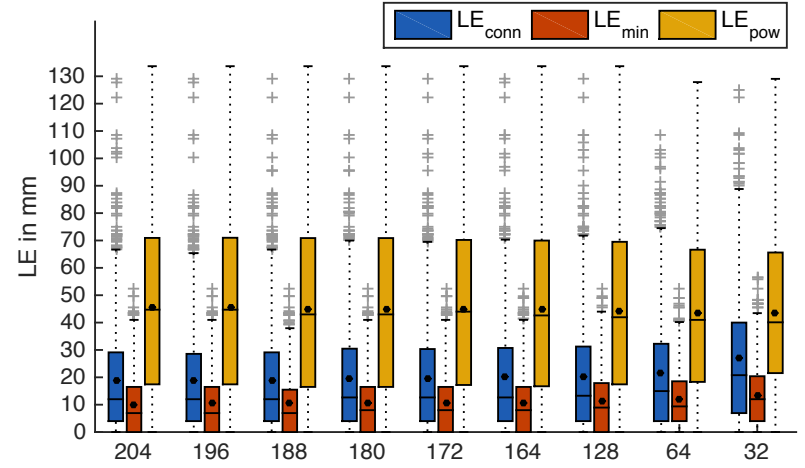

Figure 6 Overview of (i) the localization errors (LE) of the SOZ estimated by connectivity analysis, (ii) the LEs of the source with the highest power, and (iii) the LEs of the closest selected source for the different electrode setups for the simulated data. The distribution of the LEs is shown as a boxplot, the dot symbolizes the mean LE, while the bar indicates the median LE 


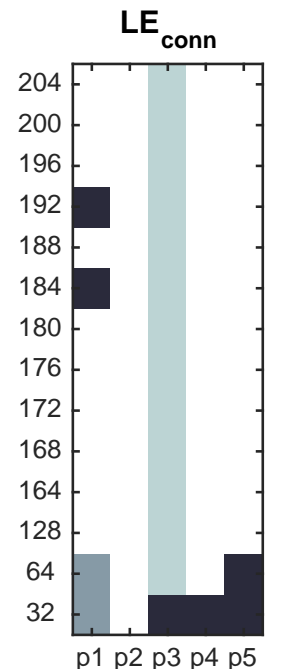

(a)

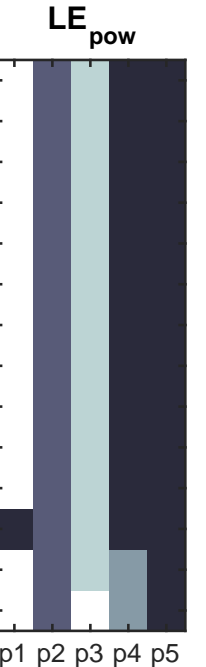

(b)

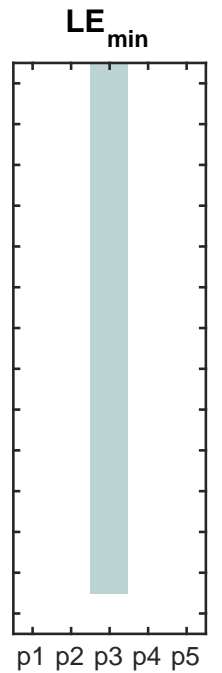

(c)

Figure 7 Overview of (a) the localization errors (LE) of the SOZ estimated by connectivity analysis, (b) the LEs of the source with the highest power, and (c) the LEs of the closest selected source for the different electrode setups for the 5 patients. 


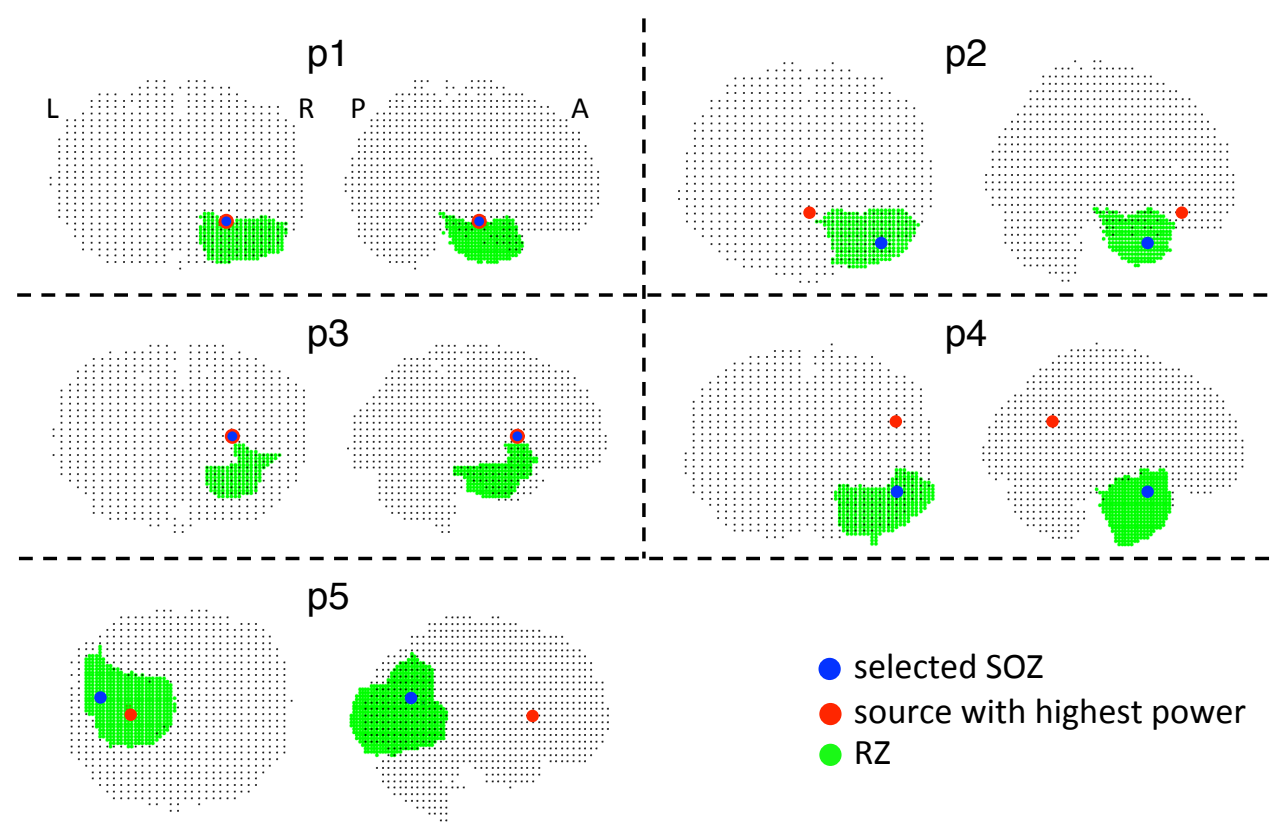

Figure 8 The selected SOZ based on the presented method (blue), the source with the highest power (red) and the resected zone (green) in the solution space for every patient, using the 204 electrode setup. $\mathrm{L}=$ left, $\mathrm{R}=$ right, $\mathrm{A}=$ anterior, $\mathrm{P}=$ posterior 


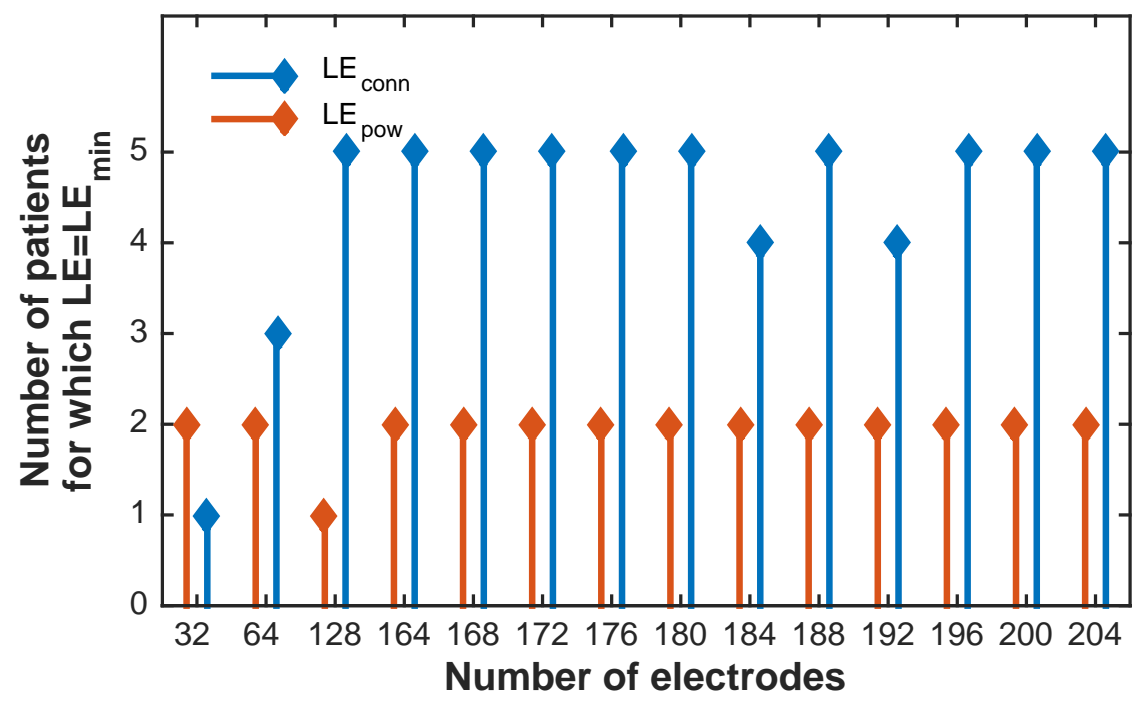

Figure 9 Representation of the number of patients for who the lower limit of error is achieved for each of the methods for all electrode setups, i.e. in how many patients does the source with the highest outdegree/the source with the highest power coincides with the source closest to the RZ for each setup 